\title{
Tropheryma whipplei
}

National Cancer Institute

\section{Source}

National Cancer Institute. Tropheryma whipplei. NCI Thesaurus. Code C86827.

A species of aerobic, Gram positive, rod shaped bacteria assigned to the phylum

Actinobacteria. This species can form filaments and has a membrane that is external to the cell wall. T. whipplei is found in soil and is a commensal organism of the oral cavity but may become pathogenic, being the causative agent of Whipple's Disease. 